\title{
Analysis of Noise Characteristics of Multichannel Systems of the Formation of Signals of Georadars with Synthesized Aperture
}

\author{
Oleg R. Kuzichkin $\mathbb{D}^{1},{ }^{1}$ Dmitriy I. Surzhik $\mathbb{D}^{2}{ }^{2}$ Gleb S. Vasiliev $\mathbb{D}^{1}{ }^{1}$ \\ Igor A. Kurilov $\mathbb{D}^{2}{ }^{2}$ and Nikolai V. Dorofeev (iD $^{2}$ \\ ${ }^{1}$ Belgorod State University, Belgorod 308015, Russia \\ ${ }^{2}$ Vladimir State University, Vladimir 600000, Russia \\ Correspondence should be addressed to Oleg R. Kuzichkin; oldolkuz@yandex.ru
}

Received 20 May 2018; Accepted 23 October 2018; Published 4 December 2018

Academic Editor: S. M. Rezaul Hasan

Copyright ( 2018 Oleg R. Kuzichkin et al. This is an open access article distributed under the Creative Commons Attribution License, which permits unrestricted use, distribution, and reproduction in any medium, provided the original work is properly cited.

\begin{abstract}
The noise characteristics of multichannel systems of forming signals based on hybrid frequency synthesizers with automatic compensation of phase distortions of direct digital synthesizers, which are used in the composition of georadars with synthesized aperture, are investigated. It is established that the phase noise of the output signals of the formers at the $1 \mathrm{kHz}$ detuning from the carrier oscillation at the output frequencies of the devices in the range from 500 to $3500 \mathrm{MHz}$ is characterized by a level of minus 100 - minus $130 \mathrm{~dB}$. In this case, the circuit of the signal former based on a hybrid frequency synthesizer with direct digital synthesizer as a reference oscillator of a phase locked loop is characterized by the worst noise characteristics but with the highest degree of autocompensation (about $13 \mathrm{~dB}$ ). Conversely, the circuit of the signal former based on a hybrid frequency synthesizer with direct digital synthesizer as a support generator of the phase-locked loop has the best phase noises level from the considered variants of devices and least degree of autocompensation (about $6 \mathrm{~dB}$ ).
\end{abstract}

\section{Introduction}

Georadar subsurface probing is one of the most effective methods of nondestructive testing. It is used for detecting, localizing, and monitoring the boundaries of mediums with different absorption coefficients, objects in these probed mediums, defects in various coatings, etc. [1-7]. One of the options for its implementation is holographic ultrawideband probing, based on the use of georadars (GPR) with a synthesized aperture [3-7]. The idea of this radar technique with respect to subsurface probing is based on the emission of ultra-wideband signals and recording the results of their reflection from objects located under the frontier of probed media having differences in the permittivity. With this type of probing, the reflected signals contain the most complete information about the properties of the inhomogeneities of the medium. The processing of reflected signals obtained as a result of successive scanning of the surface under research allows one to reconstruct the three-dimensional distribution of inhomogeneities under the interface of the probed media.

The working frequency range of modern georadars usually lies in the frequency band from 50 to $2000 \mathrm{MHz}$, which is a compromise between the depth of probing (in tens of meters) and the resolution (in tens of centimeters) $[4,5]$. However, more high-frequency georadars with an upper operating frequency above $10 \mathrm{GHz}$ are also known. An example of such systems is the products of IDS (Italy) for the mining industry, which has an operating frequency of about $17 \mathrm{GHz}$ and is intended for monitoring the dynamics of the slopes of quarries and predicting the possible occurrence of landslides in them.

In practical use of georadars, depending on the type of problems to be solved, the required probing depth and resolving power and associated with it the carrier frequency of the ultra-wideband signal are determined. In this regard, a necessary element of the subsurface probing system with a 


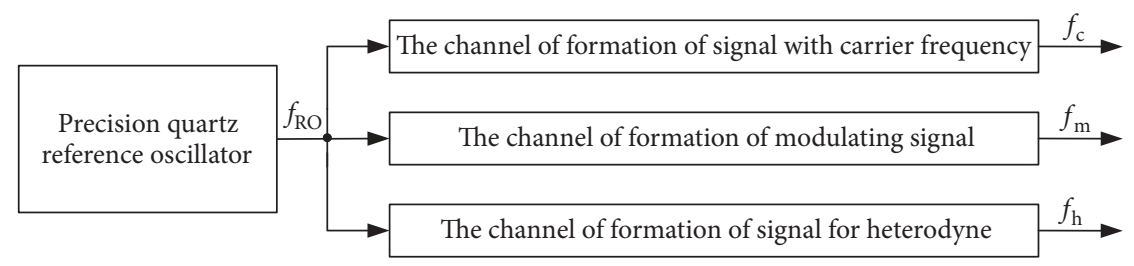

FIGURE 1: A generalized block diagram of a multichannel system the formation of signals of georadars with a synthesized aperture.

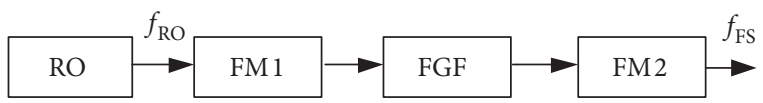

FIGURE 2: The generalized structural scheme of the channel for the formation of signals.

synthesized aperture [5] should be a multichannel system of the formation of signals that allows quick adjustment of the frequency of the output signal of the device with the required resolution and in a given frequency range. A generalized block diagram of a multichannel system the formation of signals of georadars with a synthesized aperture is shown in Figure 1.

The use of several channels of signals formation is necessary for modulation of the ultra-wideband probing signal (from two signals with a carrier $f_{\mathrm{c}}$ and modulating $f_{\mathrm{m}}$ frequencies) and generating the output signal of the heterodyne for the transceiver module with a frequency $f_{\mathrm{h}}$. In addition, to provide inphase processing of the reflected signals, the formers must ensure the coherence of the synthesized signals for each of the channels. This condition is achieved by using one common high stable source of reference frequency $f_{\mathrm{RO}}$ based on a precision quartz oscillator.

The choice of the option for constructing each of the channels of formation of signals of the GPR with synthesized aperture is an independent task that requires detailed research. Its relevance is due to a number of requirements that the signal former must provide. One of them is the allowable spectral characteristics of the formed signals, determined by the low level of discrete parasitic spectral components and phase noises. When they are achieved, the sensitivity of the receiving tract of the GPR is significantly increased and the procedure for extracting the reflected signal against the background of the existing external disturbances, usually additively added to the useful signal is simplified.

\section{Materials and Methods}

Generally, the channels for the formation of signals of various radio systems are constructed on the basis of the generalized structural scheme shown in Figure 2. It adopts the following designations: RO: reference quartz oscillator; FGF: the former of a grid of frequencies; FM1 and FM2: the frequency multipliers of the reference oscillator and the former of a grid of frequencies, respectively.

One of the promising ways to implement the channels for the formation of signals for georadars is hybrid frequency synthesis based on direct digital and indirect methods of frequency synthesis. Direct digital method of synthesis is based on the use of direct digital synthesizers (DDS), indirect-on the use of frequency synthesizers based on phase locked loops (PLL) [8-13]. In accordance with the generalized structural scheme of the channel for the formation of signals (Figure 2), the DDS performs the function of the former of a grid of frequencies, and the PLL loop is the output frequency multiplier FM2. As a frequency multiplier of the reference oscillator FM1, as a rule, transistor cascades with small multiplication factors are used.

A hybrid method of frequency synthesis makes it possible to significantly reduce the effect of the shortcomings of each of the methods used for forming signals in separately [13]. In the case under consideration (a combination of direct digital and indirect synthesis methods), the former will have improved parameters in the values of the maximum synthesized frequency and its resolving power.

Figures 3-5 show the basic block diagrams of the channels for the formation of signals based on hybrid frequency synthesizers combining direct digital and indirect methods of frequency synthesis. They reflect the main ways to turn on the DDS in the PLL loop: as a reference PLL oscillator (Figure 3), a frequency divider in the feedback loop (Figure 4) and a support oscillator of the PLL (Figure 5). The following designations are accepted on the diagram: PD: phase detector, LPF: low pass filter, VCO: voltage controlled oscillator, FD: frequency divider, and Mix: frequency mixer.

In these circuits of the channels for the formation of signals the output frequency range is provided by significant multiplication in the PLL, and a high frequency resolution is provided by the DDS. Multiplication of the frequency of the reference oscillator is necessary to achieve the maximum frequency of comparison of the phase detectors of the PLLs, which provides better noise characteristics of the synthesized signals of the formers.

In the circuit shown in Figure 4, due to the possible high frequency of the VCO and the existing limitation on the clock frequency of the DDS, its frequency predivider is used. In the scheme of a hybrid frequency synthesizer with a DDS as a support oscillator of the PLL, the division coefficient of the divider FD1 is selected based on the need to ensure equality between the comparison frequency in the phase detector and the difference frequency of the mixer output between the output frequencies of the VCO and the DDS. The frequency multiplier (FM2) in this circuit is designed to form a high clock frequency of the DDS and preserve the coherence of the device signals, which can be disrupted by the use of an additional reference frequency source for the bias circuit. The frequency divider FD2 in the feedback circuit serves to increase the output frequency of the VCO. 


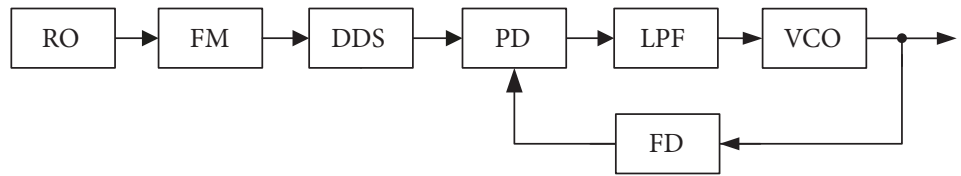

FIGURE 3: The block diagram of the hybrid frequency synthesizer with DDS as a reference PLL oscillator.

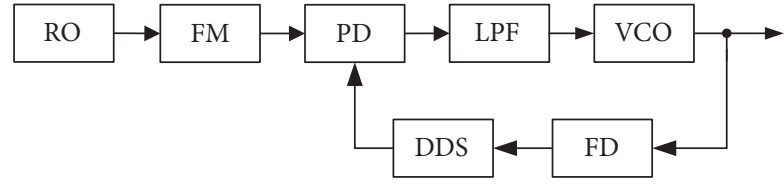

FIGURE 4: The block diagram of the hybrid frequency synthesizer with DDS as a frequency divider in the feedback loop of the PLL.

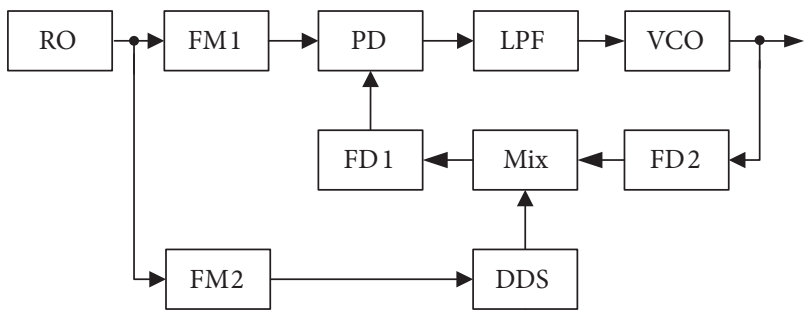

FIGURE 5: The block diagram of the hybrid frequency synthesizer with DDS as a support oscillator of the PLL.

Providing high technical parameters (speed, resolving power, and range of synthesized frequencies), the considered variants of realization of the channels for the formation of signals of georadars with a synthesized aperture based on hybrid frequency synthesizers have insufficient spectral purity of the synthesized signals. The presence in the spectrum of synthesized signals of such devices the phase noise and, what is especially important, a significant level of discrete parasitic spectral components are largely due to the contribution of the DDS.

In the output spectrum of the DDS, spectral components that appear in the time domain in the form of parasitic phase modulation are the most undesirable. These include discrete parasitic spectral components caused by truncation of the phase code and the impact of destabilizing factors, as well as noise components associated with the quantization effect and the noises of the synthesizer units [14].

An effective method of improving the spectral characteristics of signal formers that contain a DDS is to use the method of automatic compensation for phase distortions (ACPD) [15-18]. The idea of this method with respect to a DDS is based on the isolation the clock frequency with parasitic phase modulation in the spectrum of the output signal of digital-analog converter with, its comparison with the input clock frequency of the synthesizer (without phase distortions) and the formation of a control signal. This control signal is used to subsequently change the modulating voltage of the VCO in the PLL and thereby compensate for the phase distortions of the output signal of the DDS.

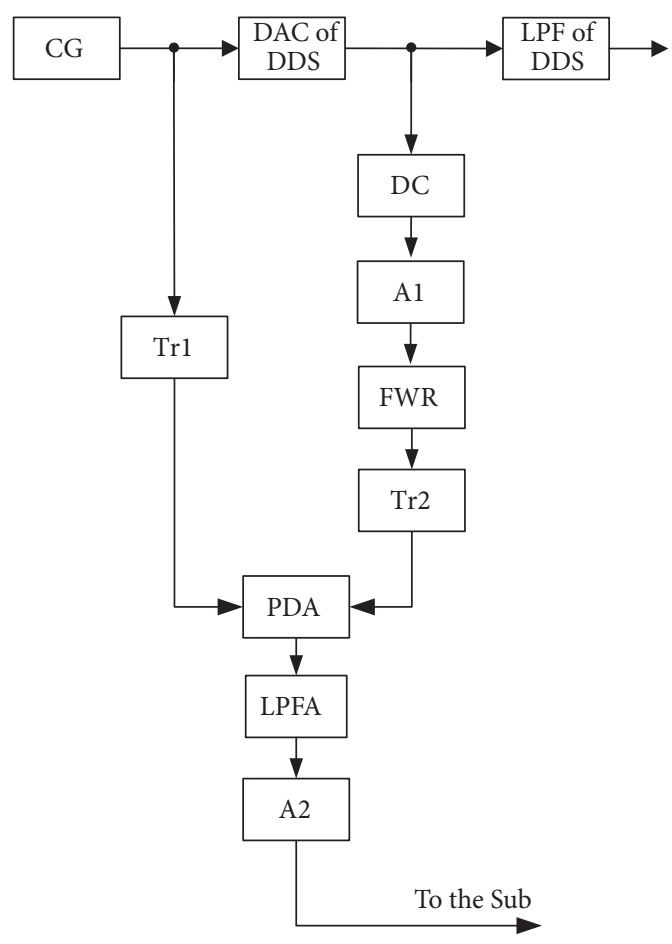

FIGURE 6: Block diagram of the control signal formation channel of the ACPD of the DDS.

The block diagram of one of the implementations of the control signal formation tract of the ACPD of the DDS is shown in Figure 6. The following designations are adopted on the diagram: CG: clock generator or source of clock frequency, DAC: digital-to-analog converter, Tr: trigger, DC: differentiating circuit, A: amplifier, FWR: full-wave rectifier, PDA: phase detector of autocompensator, LPFA: the low-pass filter of the autocompensator, and the Sub: subtractor (located between the low pass filter of the PLL loop and the VCO).

Predetection processing of signals in ACPD is aimed at eliminating the differences between the reference (clock) and informational (from the output of the DAC of the DDS) signals by amplitude and shape while maintaining phase shifts proportional to the phase distortions allocated in the control tract.

The main useful effect of the use of the ACPD circuit of the DDS in the hybrid synthesizers is to reduce the level of discrete parasitic spectral components in the output signal spectrum of the device by $10-15 \mathrm{~dB}$. However, the use of this circuit also makes it possible to reduce the level of phase noises [16-18] caused by the significant contribution of the 


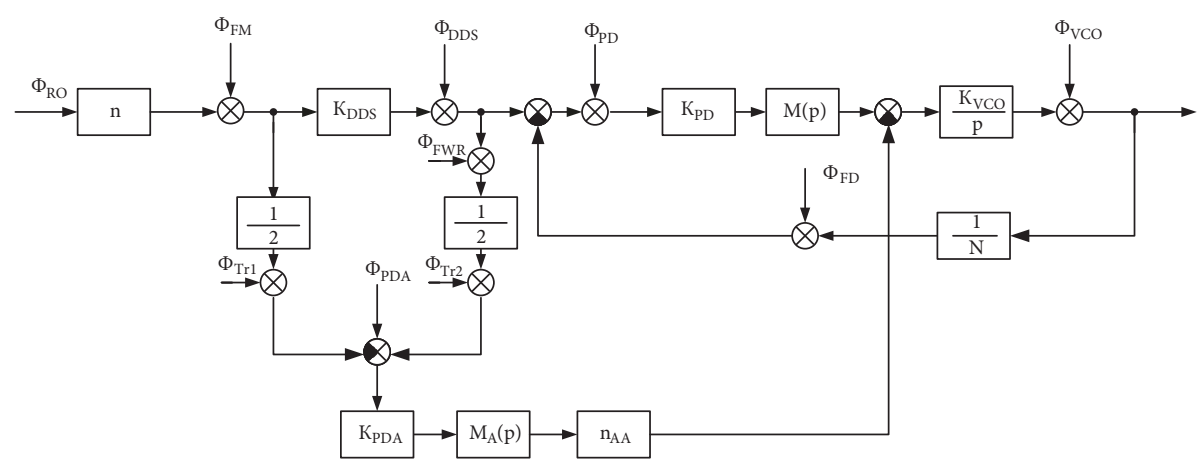

FIGURE 7: Equivalent circuit of the channel for forming signals with the DDS as a reference oscillator of the PLL with all sources of phase noises.

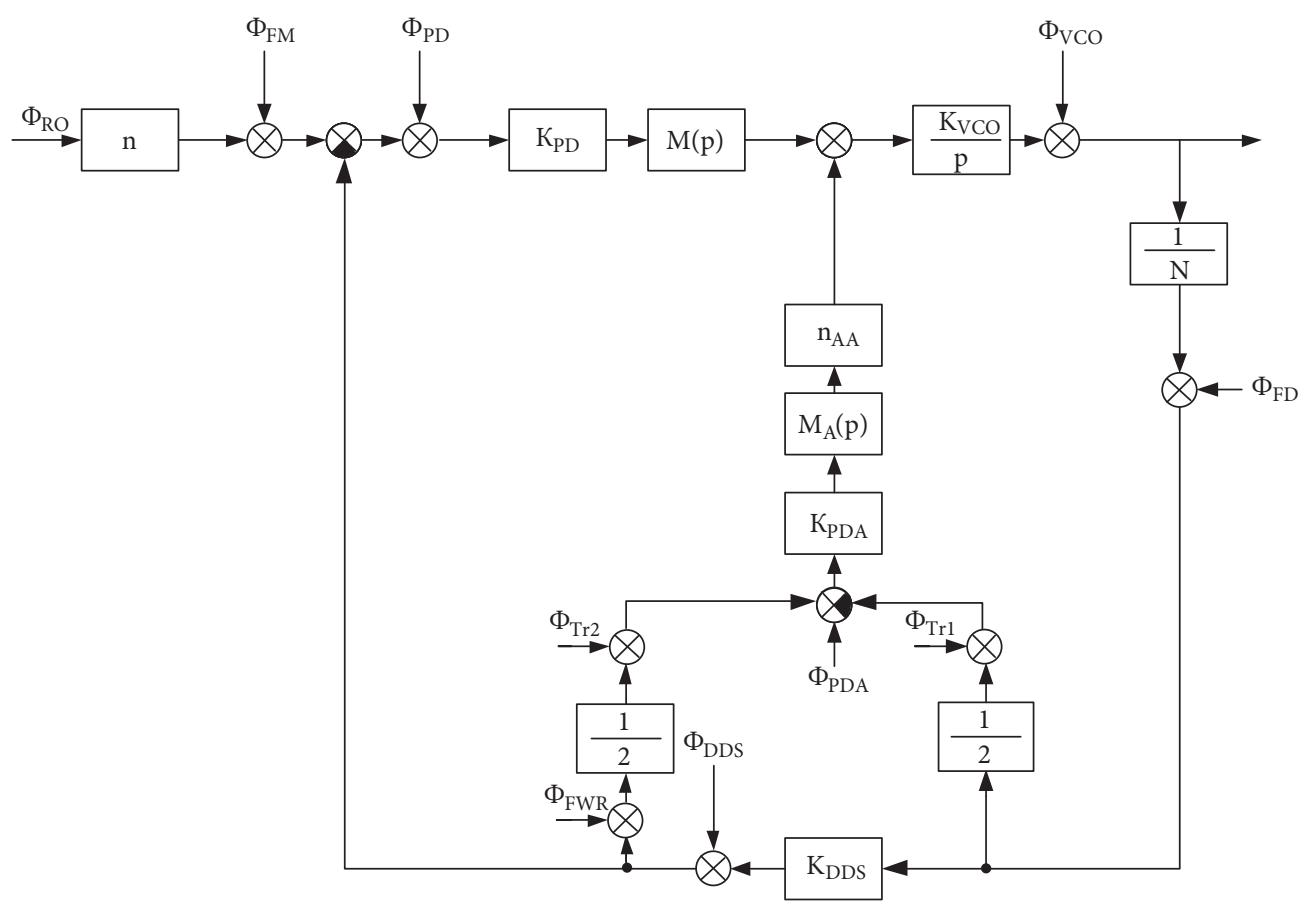

FIGURE 8: Equivalent circuit of the channel for forming signals with the DDS as a frequency divider in the feedback loop of the PLL with all sources of phase noises.

DDS which depending on its transmission coefficient and the place of inclusion in the PLL.

The main characteristic of the level of phase noises of signals is their spectral power density (SPD) $S_{\varphi}(F)$ near the carrier frequency, depending on the detuning of $F$ for different values of the output frequency [19-22]. To research the noise characteristics of the investigated hybrid frequency synthesizers (Figures 3-5) with ACPD of DDS, equivalent functional schemes with all sources of operating phase noises are compiled. In Figure 7 represents an equivalent linearized circuit of the channel for forming signals with a DDS as a reference oscillator of the PLL, in Figure 8, with the DDS as a frequency divider of the feedback loopof the PLL and in Figure 9 - with the DDS as a support oscillator of the PLL. The following designations are adopted in the schemes: $\Phi_{\mathrm{i}}$ : deviations of the phase of the respective blocks of signal forming units; $n_{\mathrm{i}}$ : the multiplication factors of the frequency multipliers of the reference oscillator; $K_{\mathrm{DDS}}$ : the transfer coefficient of the DDS; $K_{\mathrm{PD}}$ and $K_{\mathrm{PDA}}$ : the steepnesses of the detectors characteristics of the PLL and ACPD; $K_{\mathrm{VCO}}$ : the steepness of the modulation characteristic of the VCO; $N_{\mathrm{i}}$ : coefficients of divisions of the frequency dividers of the PLLs; $M(p)$ and $M_{\mathrm{A}}(p)$ are the transmission coefficients of the PLL and ACPD filters; $n_{\mathrm{AA}}$ is the amplification factor of the A2 amplifier.

On the basis of the presented equivalent circuits, mathematical models of the noise characteristics of the investigated variants of the construction of channels for the formation of signals of georadars with a synthesized aperture based on hybrid frequency synthesizers with ACPD of the DDS are obtained. It was believed that $M_{\mathrm{A}}(p)=M(p)$ and $K_{\mathrm{PDA}}=$ $K_{\mathrm{PD}}$. 


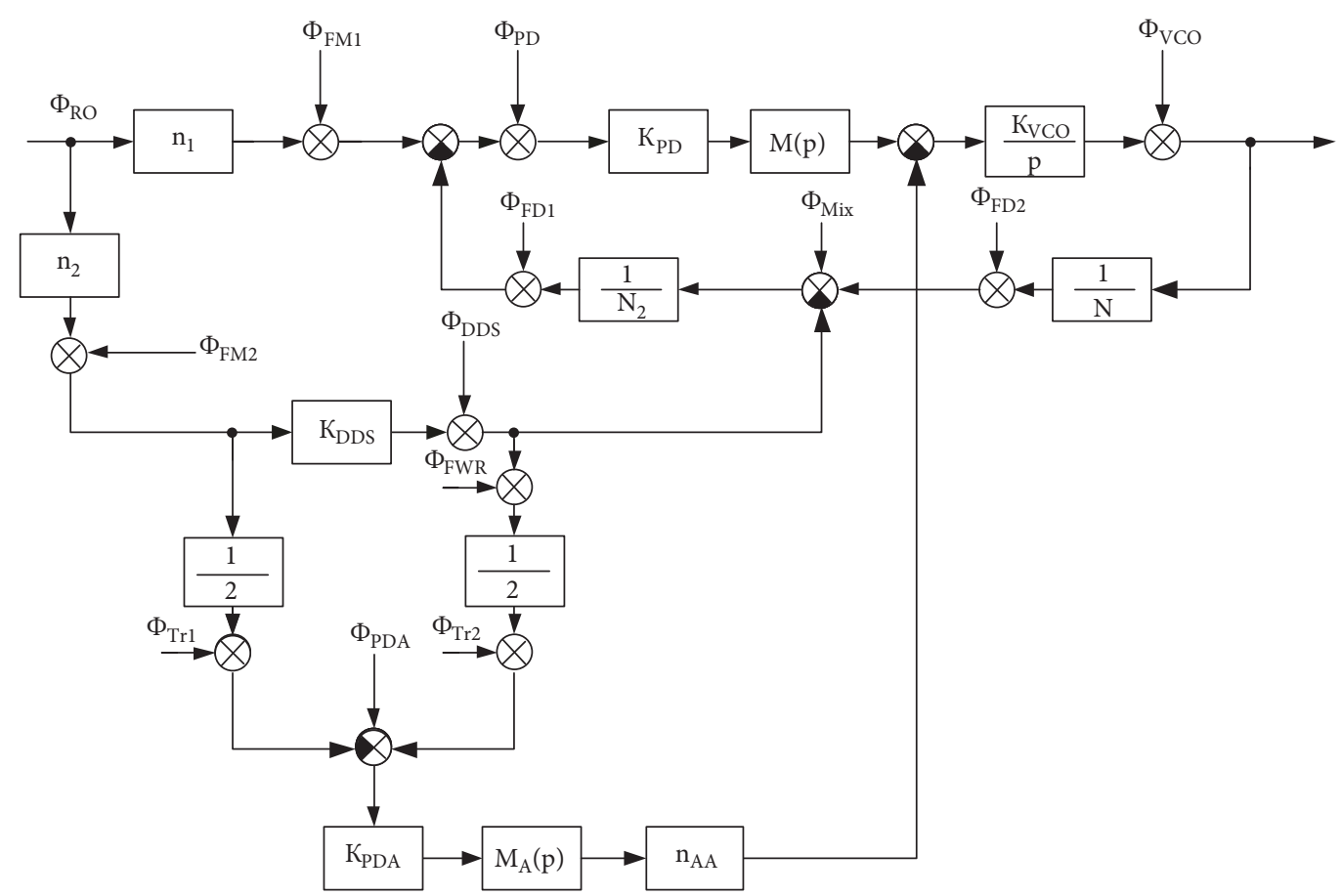

FIGURE 9: Equivalent circuit of the channel for forming signals with the DDS as a support oscillator of the PLL with all sources of phase noises.

For the circuit shown in Figure 7, the mathematical model of noise characteristics is defined as

$$
\begin{aligned}
& S_{F S 1}(F)=\left(S_{R O}(F) \cdot n^{2} \mathrm{~K}_{D D S}^{2}+S_{F M}(F) \cdot \mathrm{K}_{D D S}^{2}\right. \\
& \quad+S_{D D S}(F) \cdot\left(1-\frac{n_{A A}}{2}\right)^{2}+S_{P D}(F)+S_{F D}(F) \\
& \quad+S_{T r 1}(F) n_{A A}{ }^{2}+S_{F W R}(F)\left(\frac{n_{A A}}{2}\right)^{2}+S_{T r 2}(F) n_{A A}{ }^{2} \\
& \left.+S_{P D A}(F) \cdot n_{A A}{ }^{2}\right) \cdot\left|H_{31}(F)\right|^{2}+S_{V C O}(F)\left|H_{32}(F)\right|^{2},
\end{aligned}
$$

where $S_{\mathrm{i}}$ : the own phase noises of the respective blocks of signal former; $H_{31}(p)=H_{1}(p) N /\left(1+H_{1}(p)\right)$ : transfer function of the PLL for external noises; $H_{32}(p)=1 /(1+$ $\left.H_{1}(p)\right)$ : transfer function of the PLL for internal noises; $H_{1}(p)=M(p) K_{V C O} K_{P D} / p N$ : transfer function of the open PLL.

For the circuit shown in Figure 8, the mathematical model of noise characteristics is defined as

$$
\begin{aligned}
& S_{F S 2}(F)=\left(S_{R O}(F) n^{2}+S_{F M}(F)+S_{P D}(F)\right. \\
& +S_{F D}(F) K_{D D S}^{2}+S_{D D S}(F) \cdot\left(\frac{n_{A A}}{2}-1\right)^{2} \\
& +S_{T r 1}(F) n_{A A}{ }^{2}+S_{F W R}(F)\left(\frac{n_{A A}}{2}\right)^{2}+S_{T r 2}(F) n_{A A}{ }^{2} \\
& \left.+S_{P D A}(F) \cdot n_{A A}{ }^{2}\right) \cdot\left|H_{31}(F)\right|^{2}+S_{V C O}(F) \\
& \cdot\left|H_{32}(F)\right|^{2}
\end{aligned}
$$

where $H_{31}(p)=H_{1}(p) N /\left(1+H_{1}(p)\right) K_{D D S}, H_{32}(p)=1 /(1+$ $\left.H_{1}(p)\right)$, and $H_{1}(p)=M(p) K_{V C O} K_{P D} K_{D D S} / p N$.

For the circuit shown in Figure 9, the mathematical model of noise characteristics is defined as

$$
\begin{aligned}
& S_{F S 3}(F)=\left(S_{R O}(F) \cdot\left(n_{1}+\frac{n_{2} K_{D D S}}{N_{2}}\right)^{2}+S_{F M 1}(F)\right. \\
& +S_{P D}(F)+S_{F D}(F)+\frac{S_{M i x}(F)+S_{F D 2}(F)}{N_{2}{ }^{2}} \\
& +S_{F M 2}(F) \frac{K_{D D S}^{2}}{N_{2}{ }^{2}}+S_{D D S}(F) \cdot\left(1-\frac{n_{A A}}{2}\right)^{2} \\
& +S_{T r 1}(F) n_{A A}{ }^{2}+S_{F W R}(F)\left(\frac{n_{A A}}{2}\right)^{2}+S_{T r 2}(F) n_{A A}{ }^{2} \\
& \left.+S_{P D A}(F) \cdot n_{A A}{ }^{2}\right) \cdot\left|H_{31}(F)\right|^{2}+S_{V C O}(F) \\
& +\left|H_{32}(F)\right|^{2},
\end{aligned}
$$

where $H_{31}(p)=H_{1}(p) N_{2} N /\left(1+H_{1}(p)\right), H_{32}(p)=1 /(1+$ $\left.H_{1}(p)\right)$, and $H_{1}(p)=M(p) K_{V C O} K_{P D} / p N_{2} N$.

For a theoretical description of the level of intrinsic phase noises of the individual links of the signal formers, the SPD approximation of their phase noises is used, which greatly simplifies their investigation and allows us to compare different schemes in terms of the phase noises level. The basic models of SPD of phase noises of reference oscillators, controlled generators, phase detectors, dividers, multipliers, and mixers of frequencies are presented in [19-21], the model of SPD of phase noises of the DDS in [22]. 


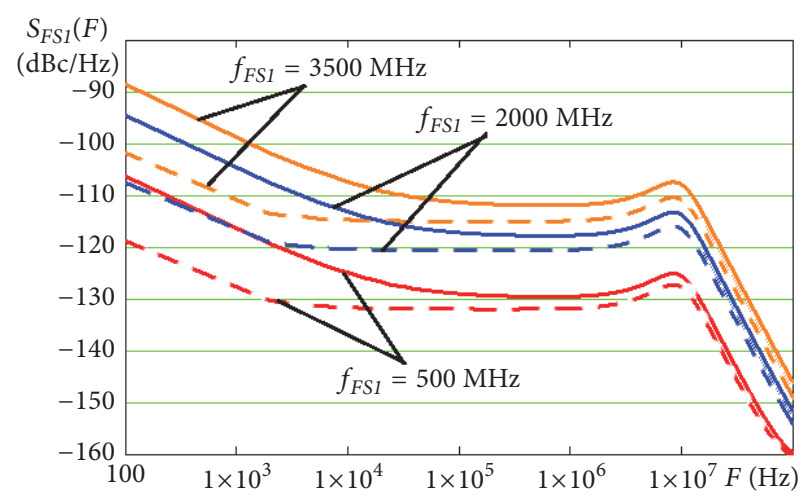

FIGURE 10: SPD of the phase noises of a hybrid frequency synthesizer with a DDS as a reference oscillator of the PLL and ACPD for output frequencies of $500 \mathrm{MHz}\left(n=3, N=5, K_{\mathrm{DDS}}=0,333\right.$, and $\left.n_{\mathrm{AA}}=1,95\right)$, $2000 \mathrm{MHz}\left(n=3, N=20, K_{\mathrm{DDS}}=0,333\right.$, and $\left.n_{\mathrm{AA}}=1,95\right)$, and 3500 $\mathrm{MHz}\left(n=3, N=35, K_{\mathrm{DDS}}=0,333\right.$, and $\left.n_{\mathrm{AA}}=1,95\right)$.

\section{Results and Discussion}

Modeling and comparative analysis of noise characteristics of the proposed variants of construction of channels for signal formation based on hybrid frequency synthesizers with ACPD of the DDS of georadars with synthesized aperture are carried out. For the circuits under consideration as the frequency values of the reference quartz oscillator were used $f_{\mathrm{RO}}=100 \mathrm{MHz}$ for the three values of the output frequency of the VCO of hybrid synthesizer $f_{\mathrm{FS}}=500,2000$, and 3500 $\mathrm{MHz}$.

As the DDS for modeling, the parameters of the AD9914 integral synthesizer with a 12-bit DAC were used. As the PLL was used the parameters of the ADF5355 synthesizer, which has the slope of the modulation characteristic of the VCO $K_{\mathrm{VCO}}$ of $15 \mathrm{MHz} / \mathrm{V}$, the steepness of the detector characteristic $K_{\mathrm{PD}}=1$, and the cutoff frequency of the LPF $f_{\mathrm{LPF}}=10 \mathrm{MHz}$.

Figure 10 shows the noise characteristics of the hybrid frequency synthesizer in the presence (dotted curves) and the absence (continuouscurves) of the ACPD circuit of the DDS as the reference oscillator of the PLL, in Figure 11 for the circuit with the DDS as a frequency divider in the feedback circuit of the PLL, and in Figure 12 for the circuit with the DDS as the support oscillator of the PLL. In this case, filters of the first order were used as filters of the PLL and the ACPD.

\section{Conclusions}

From the obtained graphical dependencies it follows that, for the considered circuits of the devices, the phase noise of the output signals at the detuning of $1 \mathrm{kHz}$ from the carrier oscillation, depending on the output frequency of the devices, is characterized by a level of minus 100 - minus $130 \mathrm{~dB}$, which is a very good indicator. It was found that, with increasing frequencies of the output signals of the devices, a proportional increase in their phase noises is observed.

The lowest level of phase noises from the proposed schemes has a hybrid frequency synthesizer with a DDS as a support oscillator of the PLL, and the largest with a DDS as

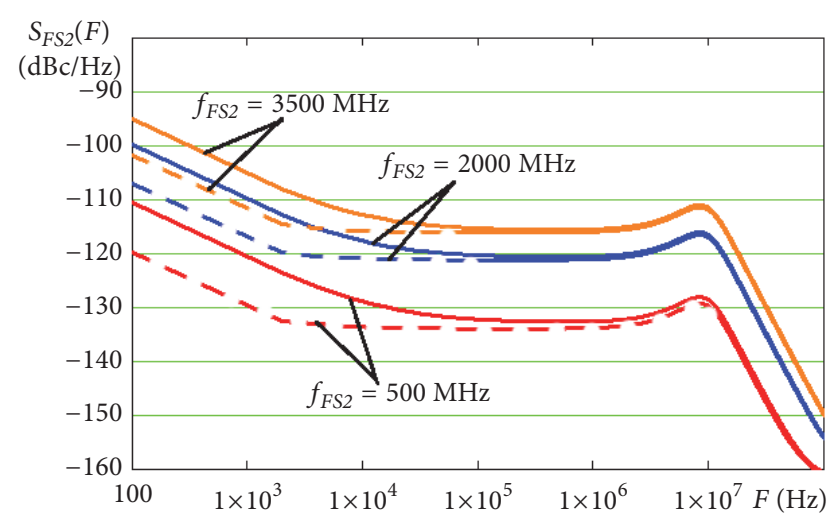

FIGURE 11: SPD of phase noises of a hybrid frequency synthesizer with a DDS as a frequency divider in the feedback loop of the PLL and the ACPDfor output frequencies of $500 \mathrm{MHz}\left(n=1, N=1, K_{\mathrm{DDS}}\right.$ $=0,2$, and $\left.n_{\mathrm{AA}}=1,95\right), 2000 \mathrm{MHz}\left(n=1, N=1, K_{\mathrm{DDS}}=0,05\right.$, and $n_{\mathrm{AA}}$ $=1,95)$, and $3500 \mathrm{MHz}\left(n=1, N=2, K_{\mathrm{DDS}}=0,057\right.$, and $\left.n_{\mathrm{AA}}=1,95\right)$.

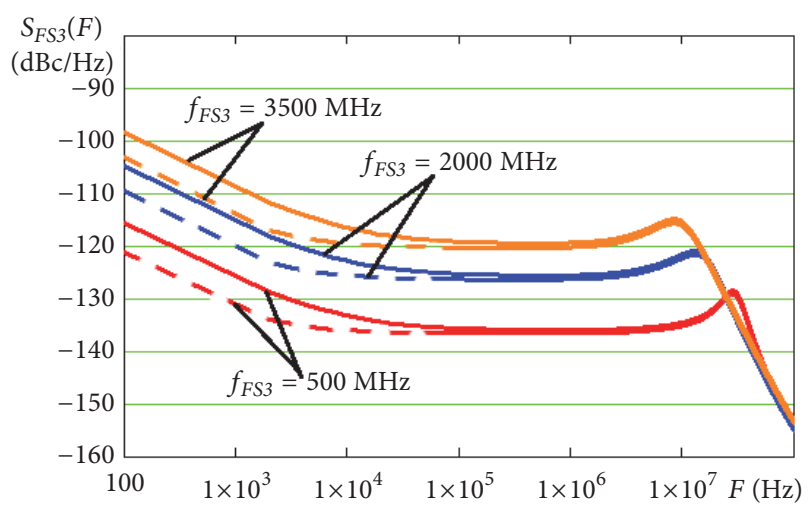

FIGURE 12: SPD of the phase noises of a hybrid frequency synthesizer with a DDS as a support oscillator of the PLL and ACPD for output frequencies of $500 \mathrm{MHz}\left(n_{1}=1, n_{2}=6, N=2, N_{2}=1, K_{\mathrm{DDS}}=0,25\right.$, and $\left.n_{\mathrm{AA}}=1,95\right), 2000 \mathrm{MHz}\left(n_{1}=1, n_{2}=6, N=8, N_{2}=1, K_{\mathrm{DDS}}=0,25\right.$, and $\left.n_{\mathrm{AA}}=1,95\right)$, and $3500 \mathrm{MHz}\left(n_{1}=1, n_{2}=6, N=14, N_{2}=1, K_{\mathrm{DDS}}\right.$ $=0,25$, and $\left.n_{\mathrm{AA}}=1,95\right)$.

a reference oscillator of the PLL. This is due to the significant differences between these circuits by the fission factor in the feedback loop of the PLL.

At the same time, the highest degree of autocompensation of phase noises from the three proposed schemes has a hybrid synthesizer with a DDS as a reference oscillator. This is due to the predominance in the given scheme of the intrinsic contribution of the DDS in the SPD of the phase noises of the device in comparison with other components of the phase noise. In addition, it is seen that, with an increase in the frequencies of the output signals, a slight decrease in the degree of autocompensation of the phase noises of the output signals is observed for all the circuitry of the signal formers.

Theoretical values of the maximum suppression of phase noises in the spectrums of output signals of the proposed variants of the construction of signals formation channels based on hybrid frequency synthesizers with a DDS as a reference oscillator of the PLL are $13 \mathrm{~dB}$, for a circuit with a 
DDS as a frequency divider the feedback circuits are $10 \mathrm{~dB}$, and for the scheme with a DDS as a support oscillator of the PLL is characterized by a value of $6 \mathrm{~dB}$ [23].

The presented results confirm the efficiency of the use of hybrid frequency synthesizers as the signal formers for georadars with synthesized aperture, as well as the method of automatic compensation to improve their noise characteristics.

\section{Data Availability}

The data used to support the findings of this study are available from the corresponding author upon request.

\section{Conflicts of Interest}

The authors declare that they have no conflicts of interest.

\section{Acknowledgments}

This work was supported by a grant of the Ministry of Education and Science of the Russian Federation (Grant no. 5.3606.2017/PCH).

\section{References}

[1] S. V. Izyumov, S. V. Druchininand, and A. S. Voznesensky, "Theory and methods of georadiolocation," The Mountain Book, p. 196, 2008.

[2] M. I. Finkelstein, V. L. Mendelsonand, and V. A. Kutev, "Radiolocation of layered earth cover," M: Sov. radio, p. 176, 1977.

[3] M. I. Finkelstein, V. A. Kutev, and V. P. Zolotarev, "Application of radar subsurface probing in engineering geology," M: Nedra, p. 128, 1986.

[4] A. Y. Grinev, "Sub-surface radar issues," M: Radio Engineering, p. 416, 2005.

[5] G. S. KondratenkovandA, "Radio broadcasting.Radar systems for remote sensing of the Earth," M: Radio Engineering, p. 368, 2005.

[6] V. S. Verba, "Radar systems of ground-based space survey," $M$ : Radio Engineering, p. 681, 2010.

[7] V. N. Antipov, "Radar stations with digital synthesis of antenna aperture," M: Radio and Communication, p. 304, 1988.

[8] J. Vankka and K. Halonen, Direct Digital Synthesizers: Theory, Design and Applications, Helsinki University of Technology, 2000.

[9] V. F. Kroupa, Phase Lock Loops and Frequency Synthesis, John Wiley \& Sons, 2003.

[10] B.-G. Goldberg, Digital Frequency Synthesis Demystified DDS and Fractional-N PLLs, LLH Technology Publishing, 1999.

[11] L. A. Belov, "Formation of stable frequencies and signals," $M$ : Publishing Center Academy, p. 224, 2005.

[12] N. P. Yampurin, E. V. Safonova, and E. B. Zhalnin, "Formation of precision frequencies and signals," Nizhny Novgorod State Technical University, p. 187, 2003.

[13] L. I. Ridiko, "DDS: direct digital frequency synthesis," Components and Technologies, vol. 7, 2001.

[14] W. Kester, "Analog-to-digital conversion," M: Technosphere, p. 1016, 2007.
[15] D. I. Surzhik, I. A. Kurilov, and G. S. Vasiliev, "Compensation of distortions of the DDS in hybrid frequency synthesizers," Radio engineering and telecommunication systems, vol. 4, no. 20, pp. 13-19, 2015

[16] D. I. Surzhik, I. A. Kurilov, O. R. Kuzichkin, G. S. Vasilyev, and S. M. Kharchuk, "Modeling the noise properties of hybrid frequency synthesizers with automatic compensation of phase noise of DDS," in Proceedings of the 2015 International Siberian Conference on Control and Communications, SIBCON 2015, Russia, May 2015.

[17] G. S. Vasilyev, O. R. Kuzichkin, I. A. Kurilov, and D. I. Surzhik, "Analysis of noise properties of hybrid frequency synthesizer with autocompensating phase noise of DDS and PLL," in Proceedings of the 2016 International Siberian Conference on Control and Communications, SIBCON 2016, Russia, May 2016.

[18] D. I. Surzhik, I. A. Kurilov, G. S. Vasilyev, and S. M. Kharchuk, "Design and mathematical modeling of hybrid frequency synthesizers with automatic compensation of DDS interferences," in Proccedings of the 2015 International Siberian Conference on Control and Communications, IEEE Catalog Number: CFP15794-CDR, 2016.

[19] A. V. Ryzhkov and V. N. Popov, "Frequency synthesizers in radio communication technology," M.: Radio and Communication, p. 264, 1991.

[20] V. Zhalud and V. Kuleshov, "Noise in semiconductor devices," M.: Sov Radio, p. 416, 1997.

[21] M. Buckingham, "Noises in electronic devices and systems," M.: Mir, p. 399, 1986.

[22] V. V. Romashov, L. V. Romashova, K. K. Khramov, A. N. Doctorov, and K. A. Yakimenko, "Simulation of noise characteristics of hybrid frequency synthesizers," Radio Engineering and Telecommunication Systems, vol. 1, pp. 5-20, 2014.

[23] D. I. Surzhik, Abstract of the Dissertation of Candidate of Technical Sciences "Direct Digital Synthesizers with Automatic Compensation of Phase Distortions" [dissertation, thesis], Vladimir state university, 2016. 


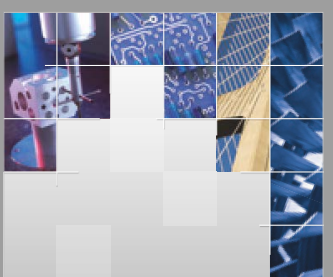

\section{Enfincering}
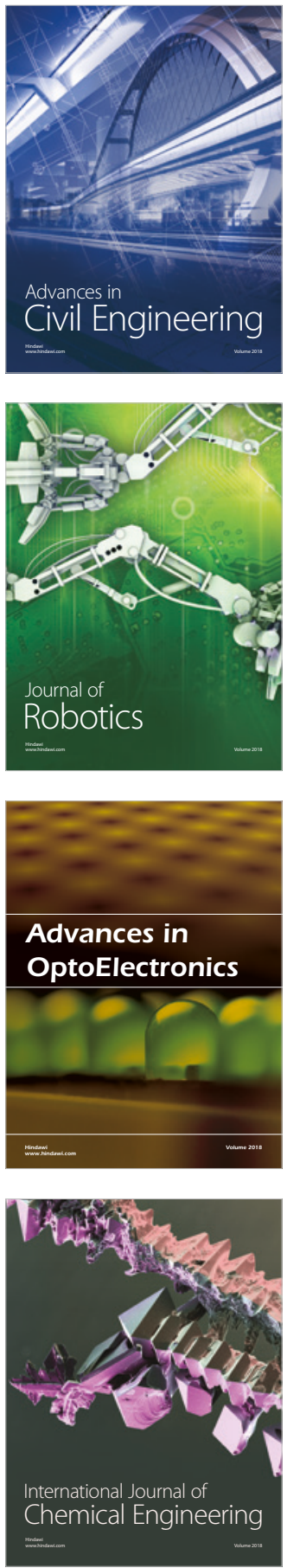

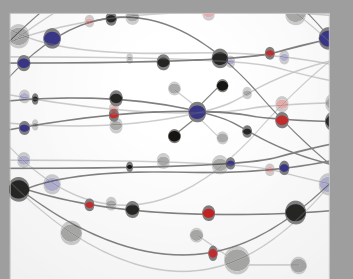

\section{Rotating \\ Machinery}

The Scientific World Journal

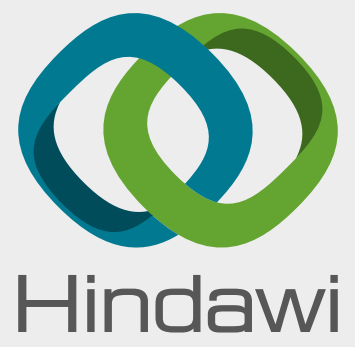

Submit your manuscripts at

www.hindawi.com
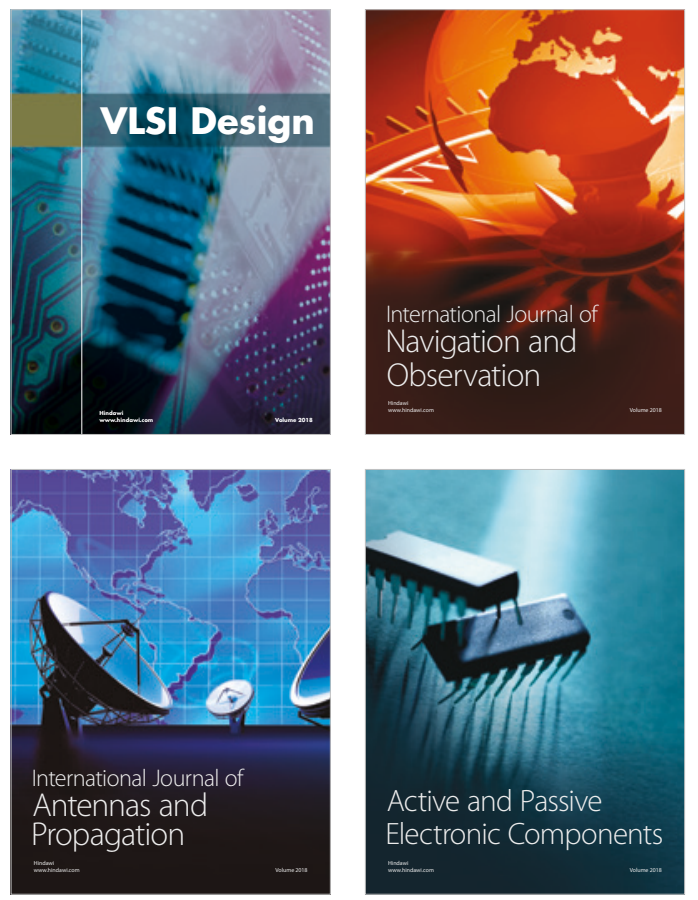
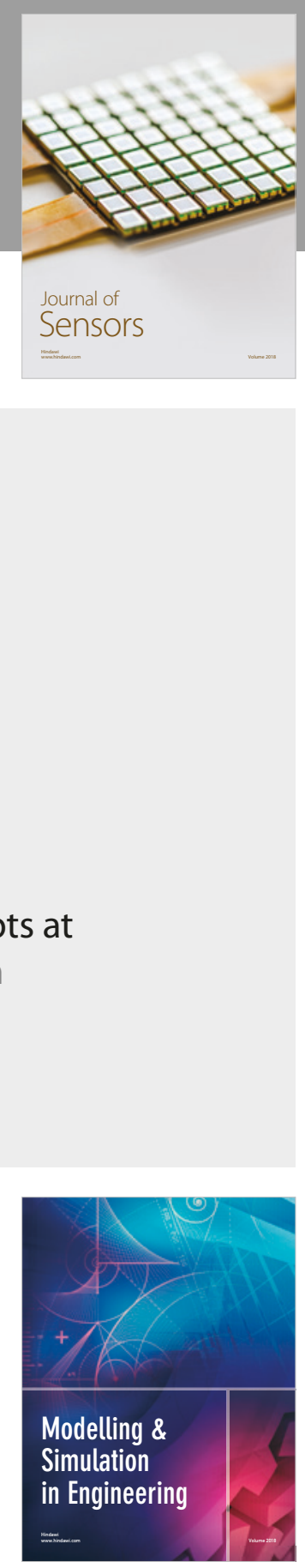

\section{Advances \\ Multimedia}
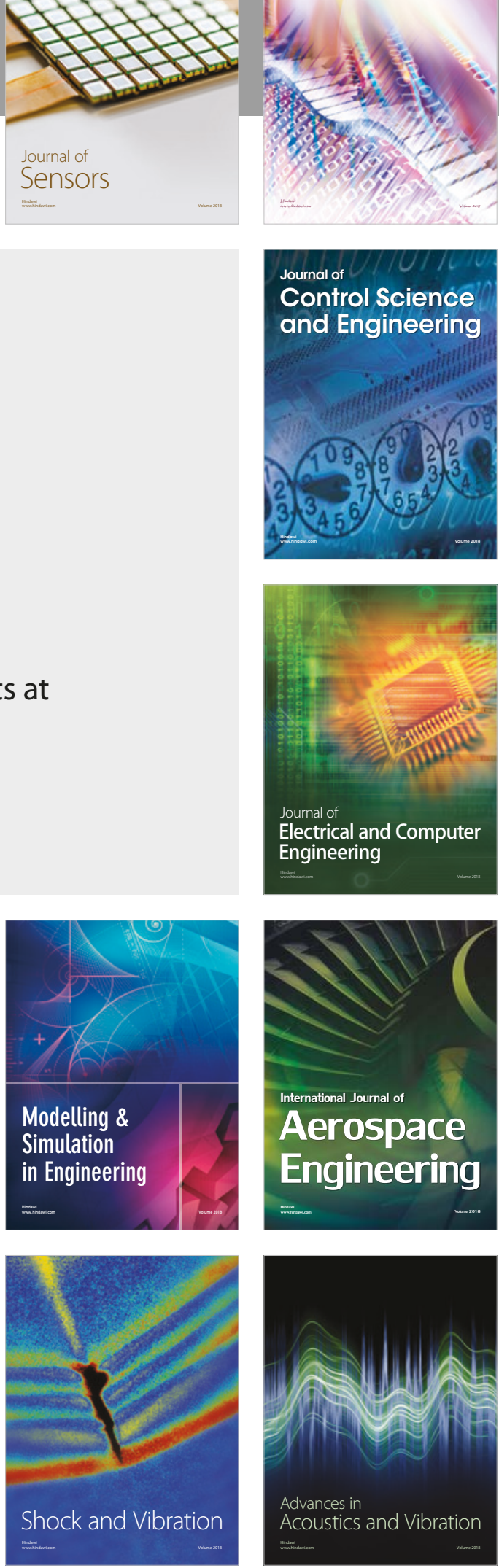